\title{
An assessment of the most reliable method to estimate the sagittal alignment of the cervical spine: analysis of a prospective cohort of 138 cases
}

\author{
Roland D. Donk, MD, ${ }^{1}$ Michael G. Fehlings, MD, PhD, FRCSC, ${ }^{2}$ Wim I. M. Verhagen, MD, PhD, ${ }^{3}$ \\ Hisse Arnts, MD, ${ }^{4}$ Hans Groenewoud, MSc, ${ }^{5}$ André L. M. Verbeek, MD, PhD, ${ }^{5}$ and \\ Ronald H. M. A. Bartels, MD, PhD ${ }^{4}$
}

${ }^{1}$ Department of Orthopedic Surgery, Via Sana Clinics, Mill; ' ${ }^{3}$ Department of Neurology, Canisius Wilhelmina Hospital, Nijmegen; and Departments of ${ }^{4}$ Neurosurgery and ${ }^{5} \mathrm{Health}$ Evidence, Radboud University Medical Center, Nijmegen, The Netherlands; and 2Division of Neurosurgery and Spinal Program, Department of Surgery, University of Toronto, Ontario, Canada

OBJECTIVE Although there is increasing recognition of the importance of cervical spinal sagittal balance, there is a lack of consensus as to the optimal method to accurately assess the cervical sagittal alignment. Cervical alignment is important for surgical decision making. Sagittal balance of the cervical spine is generally assessed using one of two methods; namely, measuring the angle between C-2 and C-7, and drawing a line between C-2 and C-7. Here, the best method to assess sagittal alignment of the cervical spine is investigated.

METHODS Data from 138 patients enrolled in a randomized controlled trial (Procon) were analyzed. Two investigators independently measured the angle between C-2 and C-7 by using Harrison's posterior tangent method, and also estimated the shape of the sagittal curve by using a modified Toyama method. The mean angles of each quantitative assessment of the sagittal alignment were calculated and the results were compared. The interrater reliability for both methods was estimated using Cronbach's alpha.

RESULTS For both methods the interrater reliability was high: for the posterior tangent method it was 0.907 and for the modified Toyama technique it was 0.984 . For a lordotic cervical spine, defined by the modified Toyama method, the mean angle (defined by Harrison's posterior tangent method) was $23.4^{\circ} \pm 9.9^{\circ}$ (range $0.4^{\circ}-52.4^{\circ}$ ), for a kyphotic cervical spine it was $-2.2^{\circ} \pm 9.2^{\circ}$ (range $-16.1^{\circ}$ to $16.9^{\circ}$ ), and for a straight cervical spine it was $10.5^{\circ} \pm 8.2^{\circ}$ (range $-11^{\circ}$ to $36^{\circ}$ ). CONCLUSIONS An absolute measurement of the angle between C-2 and C-7 does not unequivocally define the sagittal cervical alignment. As can be seen from the minimum and maximum values, even a positive angle between $\mathrm{C}-2$ and C-7 could be present in a kyphotic spine. For this purpose, the modified Toyama method (drawing a line from the posterior inferior part of the vertebral body of C-2 to the posterior upper part of the vertebral body of C-7 without any measurements) is a better tool for a global assessment of cervical sagittal alignment.

Clinical trial registration no.: ISRCTN41681847 (https://www.isrctn.com)

https://thejns.org/doi/abs/10.3171/2016.10.SPINE16632

KEY WORDS cervical spine; sagittal alignment; lordosis; kyphosis

$\mathrm{S}$ AGITTAL alignment of the cervical spine is a very important consideration when undertaking surgical interventions. ${ }^{19}$ It is one of the critical factors that influence the decision for either an anterior or a posterior approach. Furthermore, lordosis is the natural shape, and the restoration of lordosis is a goal of most surgeries. Indeed, increasing evidence suggests that neurological out- comes, quality of life, and the rate of adjacent-segment degeneration are optimized with establishment of cervical lordosis.., 19

Recently, more attention has been paid to global spinal balance. ${ }^{6,7}$ Parameters like T-1 slope and sagittal vertical axis (SVA) have been introduced. These and other parameters are quantified in angles and distance (in mil- 

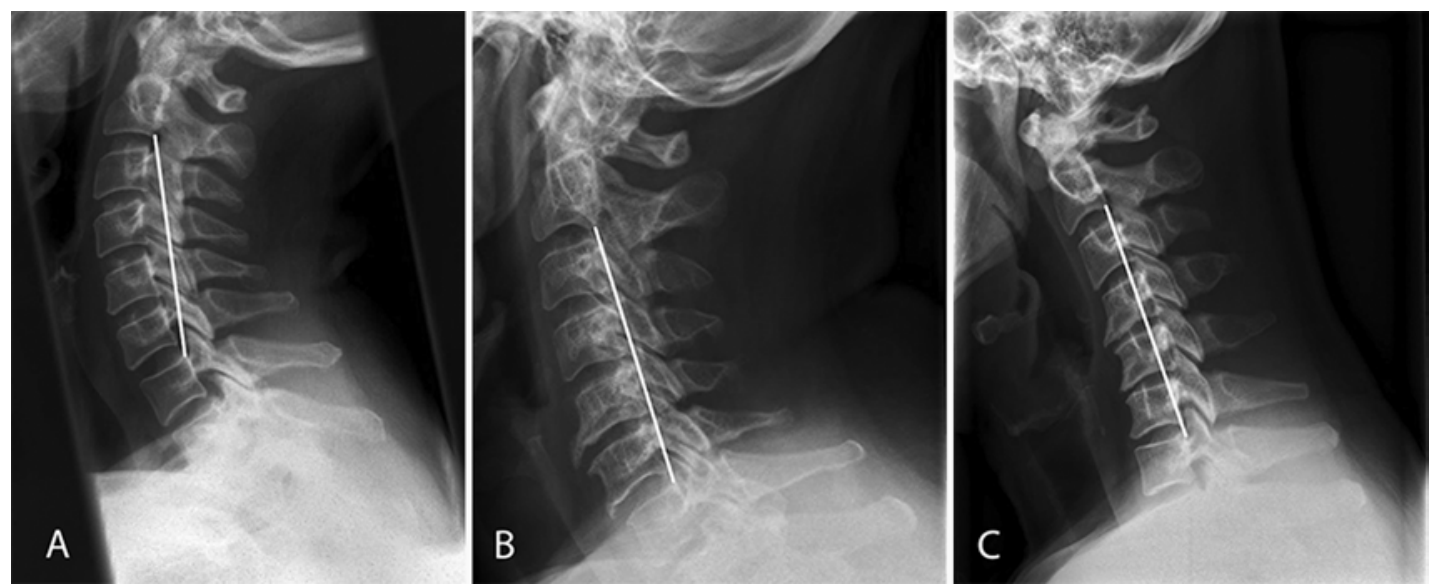

FIG. 1. Radiographs showing a modification of the method proposed by Toyama et al. A cervical lordosis $(\mathbf{A})$ is represented by the location of the vertebral bodies being anterior to the line drawn from C-2 to C-7; a straight spine (B) in which the posterior parts of the vertebral bodies are on that line; and a kyphotic spine $(\mathbf{C})$ with the posterior parts of the vertebral bodies projecting posteriorly from that line.

limeters). ${ }^{8,11,12}$ To optimize and evaluate surgical treatment of cervical pathology requires not only a quantification of different parameters, but also an assessment of the sagittal cervical alignment.

Although its importance is obvious, little attention has been given to the best way to estimate the sagittal shape of the cervical spine. Generally 2 methods exist: 1) measuring the angle between C-2 and C-7, and 2) drawing a line between $\mathrm{C}-2$ and $\mathrm{C}-7 ., 20$ For the measurement of the angle, 2 methods are generally used. One is the Cobb angle method, and the other is the posterior tangent method. Harrison et al. compared these 2 techniques and concluded that although both methods were reliable, the posterior tangent method better defines the changes in angular alignment between C-2 and C-7. ${ }^{10}$ However, the concern remains that a given value of the angle measured by the posterior tangent method does not always describe the shape of the cervical spine correctly. A comparison between a quantitative assessment of the sagittal alignment of the cervical spine with the actual angle measured by the posterior tangent method has never been performed. A clear definition of the shape of the cervical spine is of interest for clinical use, but also for research purposes. In this study we compared the quantification of the angle between $\mathrm{C}-2$ and C-7 by using Harrison's posterior tangent method, with a quantitative assessment of the curve performed using the modified Toyama method.

\section{Methods}

The study was approved by the ethics board at $\mathrm{CMO}$ Arnhem-Nijmegen, and has been conducted in accordance with the World Medical Association Declaration of Helsinki. Informed consent was obtained from all patients. The STROBE (Strengthening the Reporting of Observational Studies in Epidemiology) statement has been followed. ${ }^{21}$

The radiographs from a prospective cohort of 138 patients who participated in a randomized controlled trial $(\text { Procon) })^{2}$ were used. This trial was registered with the Current Controlled Trials database (https://www.isrctn. com), and its registration no. is ISRCTN41681847. In this trial, several techniques were compared: anterior cervical discectomy without fusion, anterior cervical discectomy with stand-alone cage, and anterior cervical discectomy with arthroplasty.

The preoperative upright lateral cervical radiographs and those of the last follow-up visit were reviewed for this study. Radiographs were digitized and made available using Impax ES (Agfa Web 1000 5.1, Agfa-Gevaert Group).

The curvature of the cervical spine was estimated according to a slight modification of the method proposed by Toyama et al. ${ }^{3,20}$ A line was drawn from the posterior and inferior part of the vertebral body of C-2 to the upper posterior part of the vertebral body of C-7. A lordotic curve was present if the posterior wall of the vertebral bodies of C3-6 were anterior to this line. The cervical spine was considered straight if the posterior part of the vertebral bodies of C3-6 was on that line, and it was kyphotic if the posterior part of the vertebral bodies was posteriorly projected to this line (Fig. 1). A sigmoid curve was present if the posterior part of the vertebral bodies was not on that line, but if a combination existed of posterior border of the vertebral bodies in front of the line and some behind that line.

Two investigators who were experienced in measuring angles (H.A. and R.H.M.A.B.) independently calculated the angle between $\mathrm{C}-2$ and $\mathrm{C}-7$, and they also estimated the shape of the curve. The Harrison posterior tangent method was used to calculate the angle between C-2 and C-7 (Figs. 2 and 3). ${ }^{10,18}$ The mean angle derived from Harrison's method was then calculated for lordotic, straight, and kyphotic cervical spines (as assessed by the modified Toyama method).

For statistical analysis SPSS Statistics version 22 (IBM Corp.) was used. Values are represented as the mean \pm SD (minimum-maximum) (Table 1). For interrater reliability, Cronbach's alpha was estimated for the measurements at the preoperative lateral radiographs.

\section{Results}

The mean age of the entire group at surgery was $44.3 \pm$ 7 years (range 18.3-59.8 years), and the female to male ra- 


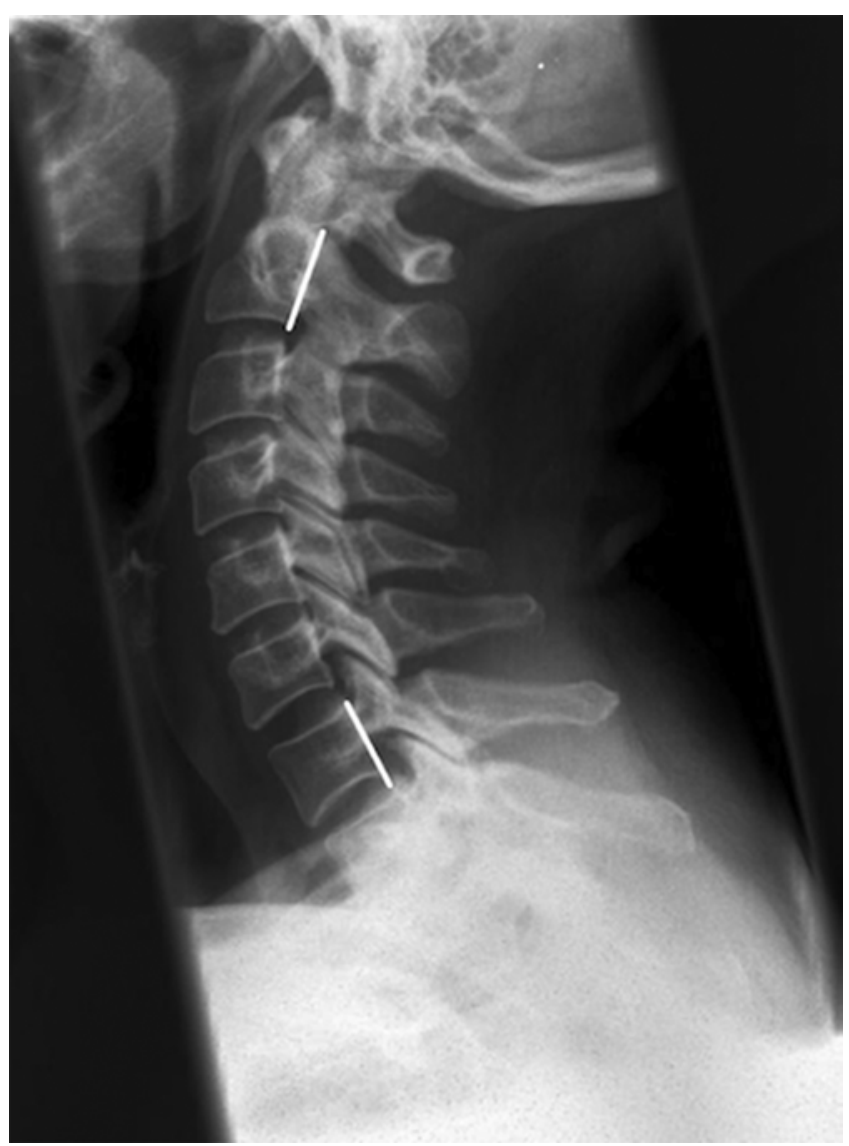

FIG. 2. Radiograph showing Harrison's posterior tangent method, in which the angle between $\mathrm{C}-2$ and C-7 is estimated by measuring the angle between the posterior bodies of $\mathrm{C}-2$ and $\mathrm{C}-7$. The lines represent the posterior bodies of $\mathrm{C}-2$ and $\mathrm{C}-7$. In this case the angle was $48.1^{\circ}$ according to the Agfa Impax software.

tio was 1:1. A total of 276 lateral radiographs of the cervical spine were retrieved and used. The mean time between the first radiograph and the radiograph obtained at the last follow-up was $25.4 \pm 18.4$ months.

Using the modified Toyoma method, lordosis was found on 128 radiographs, kyphosis on 27, and a straight spine on 121 (Table 1). A sigmoid curve was not found. Comparing the pre- and postoperative radiographs, none of the lordotic or straight cervical spines became kyphotic. Half of the kyphotic spines remained kyphotic, whereas the remaining ones were either straight or lordotic.

For lordosis the mean angle was $23.4^{\circ} \pm 9.9^{\circ}$ (range $0.4^{\circ}-52.4^{\circ}$ ), for kyphosis it was $-2.2^{\circ} \pm 9.2^{\circ}$ (range $-16.1^{\circ}$ to $16.9^{\circ}$ ), and for a straight cervical spine it was $10.5^{\circ} \pm$ $8.2^{\circ}$ (range $-10.6^{\circ}$ to $35.9^{\circ}$ ). An example of a kyphotic cervical spine and a positive angle between $\mathrm{C}-2$ and C-7 is shown in Fig. 3. The Cronbach's alpha value was 0.907 for the measurements for the angle $\mathrm{C} 2-7$, and for the modified Toyama method it was 0.984 .

Based on these data, we calculated the sample size needed and also the power based on the current sample size according to the method proposed by Bonnet and Wright. ${ }^{5}$ Considering the modified Toyama method resulting in 1 question with 3 options (lordotic, straight, or kyphotic) and assuming an expected intrarater reliability of

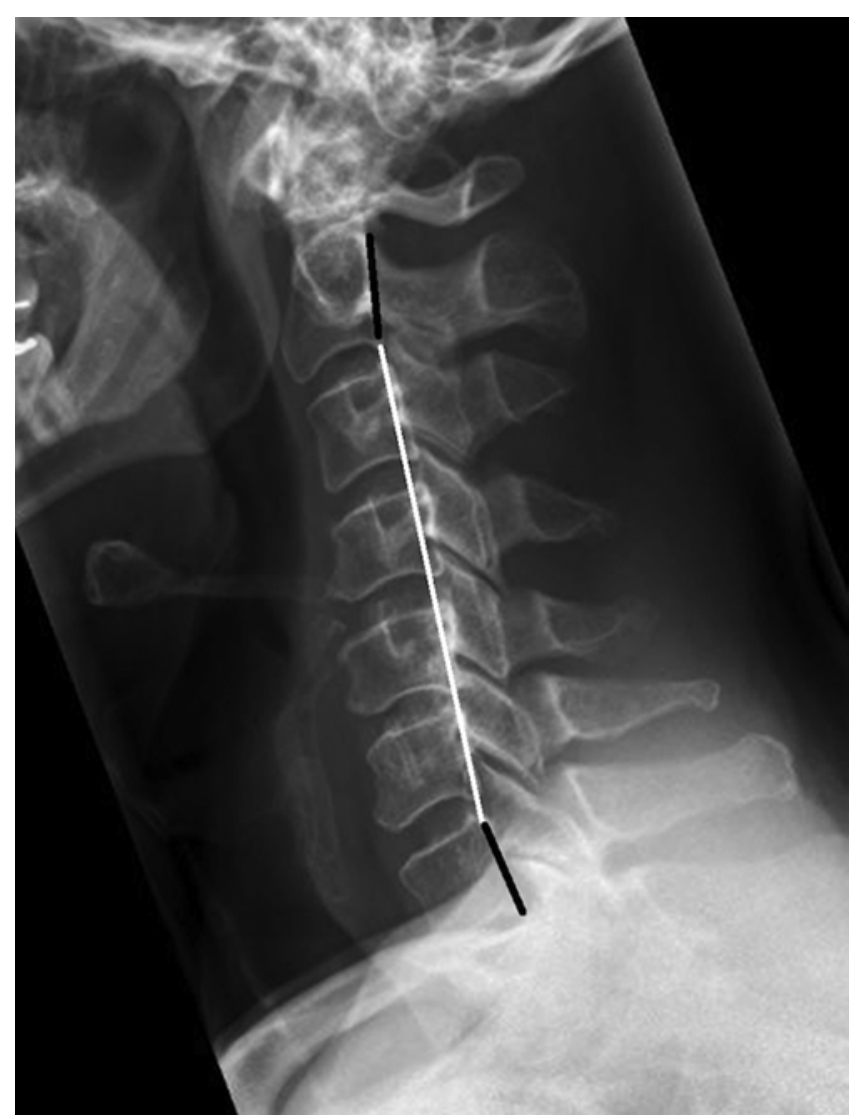

FIG. 3. Lateral upright radiograph of the cervical spine depicting a kyphotic spine measured with the modified Toyama approach, but with a positive angle measured by Harrison's posterior tangent method $\left(15.8^{\circ}\right)$.

at least $0.8, \alpha=0.05$, and a power of $80 \%$, the sample size needed would be 21 . Of note, the power of the results in a group of 276 is $100 \%$.

\section{Discussion}

This study clearly disclosed the discrepancy between the absolute values of the angle between $\mathrm{C}-2$ and $\mathrm{C}-7$ and the quantification of the cervical sagittal alignment expressed as lordotic, straight, or kyphotic. The modified Toyama method has been shown to be reliable and easy to assess. In daily practice and for the individual patient, it is of utmost importance to offer the best approach if surgery is needed.

Recently, global spinal alignment has been the subject of many investigations. As a part of these investigations attention has been paid to quantification of cervical alignment by using parameters such as cervical SVA, cervical lordosis, and T-1 slope. A correlation has been found between these parameters and health-related quality of life. ${ }^{1,13}$ The effect of surgical correction of thoracic or lumbar deformities is well known, and predictive models have even been developed..$^{14,16,17}$ In these studies the assessment of the alignment of the cervical spine was never addressed. A possible explanation is that in the case of deformity correction surgery, one of the goals is to maintain horizontal gaze while preserving the possibility to flex in the cervicothoracic region to allow walking without stumbling. ${ }^{6}$ The 
TABLE 1. Values of the angle between C-2 and C-7 as measured by the posterior tangent method in relation to categorization of lordotic, straight, or kyphotic sagittal alignment of the cervical spine

\begin{tabular}{lrrr}
\hline \multirow{2}{*}{ Angle } & \multicolumn{3}{c}{ Sagittal Alignment $\left(^{\circ}\right)$} \\
\cline { 2 - 4 } & Lordosis & Straight & Kyphosis \\
\hline C2-7 posterior tangent method & & & \\
\hline Mean & 23.4 & 10.5 & -2.2 \\
\hline SD & 9.9 & 8.2 & 9.2 \\
\hline Minimum & 0.4 & -10.6 & -16.1 \\
\hline Maximum & 52.4 & 35.9 & 16.9 \\
\hline Total no. of investigations & 128 & 121 & 27 \\
\hline
\end{tabular}

shape of the cervical spine is less important in these cases than the other quantitative measurements. Furthermore, the regional curves have a wide variation in undulation, whereas the measurements for global sagittal balance are maintained in a narrower range. ${ }^{13}$

We fully endorse the importance of global spinal alignment. However, a major shortcoming of the quantification methods mentioned above is that the actual alignment is not assessed. For example, a C2-7 SVA of $15 \mathrm{~mm}$ does not automatically mean a kyphotic spine or otherwise. For most prevalent diseases like cervical spondylotic myelopathy, the sagittal alignment is one of the factors that determines the optimal surgical approach. Sagittal alignment is of utmost importance for surgical planning and for guiding decisions on whether to offer the patient an anterior, posterior, or combined approach.

It was clearly shown that the absolute angle between C-2 and C-7 calculated using a technique that accurately depicts the cervical angle, locally and overall, ${ }^{10}$ did not reflect the alignment of the cervical spine. The angle of the vertebral body of C-2 and C-7 can be nearly zero or even positive, indicating a straight or even a lordotic spine, whereas the vertebral bodies in between (C3-6) contributed to a true cervical kyphosis.

For scientific but also practical purposes a clear and reproducible method for estimation of the cervical sagittal alignment is warranted. Toyama et al. described a method in which a line is drawn from the posterior inferior part of the vertebral body of C-2 to the upper posterior edge of the vertebral body of C-7. Then, distances between the line and the posterior border of the vertebral body should be measured, and if a certain distance $(>2 \mathrm{~mm})$ is reached, a lordotic, kyphotic, or sigmoid curve is present. ${ }^{20}$ Measuring these distances is prone to error and is not very convenient, even in these times of digitization.

Benzel also described a method in which a line was drawn between the posterior inferior part of the vertebral body of C-2 to the lower posterior edge of the vertebral body of C-7. ${ }^{4}$ This can be troublesome because C-7 is very often not completely shown on a lateral radiograph. We modified the method of Toyama et al. by using the same line without the measurements. ${ }^{3}$ If the posterior wall of the vertebral bodies of C3-6 were in front of that line cervical lordosis existed, if they were on that line it was a straight spine, and if they were behind it we called it kyphosis.
Another method involves estimating the angle in the sagittal plane between C-2 and C-7. Compared with the Cobb method the posterior tangent method was more accurate.$^{10}$ It is a general belief that a positive angle corresponds with a lordotic curve. The results of this study counter this belief.

This can be explained by the fact that the measurement between C-2 and C-7 is reflecting the angle between these 2 vertebral bodies. The position of these bodies is more or less independent of that of the intermediate vertebral bodies. For example, the posterior walls of C3-7 can be exactly collinear, but if $\mathrm{C}-2$ is angulated posteriorly the angle between C-2 and C-7 can be very positive, whereas the line drawn by the modified Toyama method projects exactly over the posterior wall of the intermediate vertebral bodies. In this way, a positive angle in the posterior tangent method is combined with a straight cervical spine. This also holds true for a kyphotic position of the vertebral bodies of C-3, C-4, C-5, and C-6, with compensation at $\mathrm{C} 2-3$ and C6-7. Locally at $\mathrm{C} 2-3$ and $\mathrm{C} 6-7$ the angles will be very positive, but between $\mathrm{C}-2$ and $\mathrm{C}-7$ these can still be positive, suggesting lordosis, whereas according to values obtained using the modified Toyama method an overall kyphotic curve is present. In other words, the posterior tangent method derives all its information locally at the end points and infers a conclusion about the entire cervical region, whereas the modified Toyama method obtains information from each segment.

Considering the high interrater reliability, both the posterior tangent method and the modified Toyama method were very useful. However, the description of the curvature of the cervical spine is more reliably represented by the modified Toyama method. With the posterior tangent method the actual curvature between C-2 and C-7 was not accurately described; e.g., in a kyphotic curve assessed by the modified Toyama method a positive angle could still be measured between C-2 and C-7.

This has practical implications. In each patient this convenient and reproducible method will facilitate the decision whether to perform surgery anteriorly or posteriorly. Also, in research the description of the curve according to this method is more reliable than the absolute value of the angle. Because this method only assesses the shape of the cervical spine without quantification, future investigations will be necessary that will facilitate its introduction in global spinal alignment measures. The combination of an assessment of the alignment of the cervical spine with a quantitative measure would be of great benefit for the comparison of various surgical approaches, for outcome research, and for educational purposes. One suggestion might be introducing it as a modifier in combination with the C2-7 SVA.

It could be argued that the results of this study were biased because the patients were participants in a randomized controlled trial (Procon). This might introduce selection bias. For example, the population was relatively young although the mean age resembled that of patients suffering from symptomatic cervical degenerative disc disease. Because the cervical alignment was not an inclusion or exclusion criterion, the relatively few patients with kyphosis in this series (9.7\%) might raise some discussion. However, 
this number also represented the normal prevalence. ${ }^{15}$ The strength of the study will not be influenced by the unevenly distributed sample size, because the sample size was much larger than needed. Furthermore, the methods we investigated can be used in any patient with any cervical curve. The high intrarater reliability in distinguishing the kyphotic and straight shape, and the post hoc power analysis of $100 \%$ provide a level of confidence in our conclusions. Also, although the method is really simple to assess and has a high intrarater reliability, we acknowledge that widespread application will reduce that reliability.

\section{Conclusions}

For practical purposes, the modified Toyama method performed by drawing a line from the posterior inferior part of the vertebral body of C-2 to the posterior upper part of the vertebral body of C-7 without any measurements is accurate, straightforward, and reliable for quantification of the sagittal alignment of the cervical spine. This technique can be readily and easily incorporated into preoperative surgical planning.

\section{References}

1. Ames CP, Blondel B, Scheer JK, Schwab FJ, Le Huec JC, Massicotte EM, et al: Cervical radiographical alignment: comprehensive assessment techniques and potential importance in cervical myelopathy. Spine (Phila Pa 1976) 38 (22 Suppl 1):S149-S160, 2013

2. Bartels RH, Donk R, van der Wilt GJ, Grotenhuis JA, Venderink D: Design of the PROCON trial: a prospective, randomized multi-center study comparing cervical anterior discectomy without fusion, with fusion or with arthroplasty. BMC Musculoskelet Disord 7:85, 2006

3. Bartels RH, Donk RD, Feuth T: Subsidence of stand-alone cervical carbon fiber cages. Neurosurgery 58:502-508, 2006

4. Benzel EC: Biomechanics of Spine Stabilization. Rolling Meadows, IL: AANS, 2001, p 85

5. Bonnet DG, Wright TA: Cronbach's alpha reliability: interval estimation, hypothesis testing, and sample size planning. J Organ Behav 36:3-15, 2015

6. Diebo BG, Challier V, Henry JK, Oren JH, Spiegel MA, Vira $\mathrm{S}$, et al: Predicting cervical alignment required to maintain horizontal gaze based on global spinal alignment. Spine (Phila Pa 1976) 41:1795-1800, 2016

7. Diebo BG, Oren JH, Challier V, Lafage R, Ferrero E, Liu S, et al: Global sagittal axis: a step toward full-body assessment of sagittal plane deformity in the human body. J Neurosurg Spine 25:494-499, 2016

8. Gillis CC, Kaszuba MC, Traynelis VC: Cervical radiographic parameters in 1- and 2-level anterior cervical discectomy and fusion. J Neurosurg Spine 25:421-429, 2016

9. Hansen MA, Kim HJ, Van Alstyne EM, Skelly AC, Fehlings MG: Does postsurgical cervical deformity affect the risk of cervical adjacent segment pathology? A systematic review. Spine (Phila Pa 1976) 37 (22 Suppl):S75-S84, 2012

10. Harrison DE, Harrison DD, Cailliet R, Troyanovich SJ, Janik TJ, Holland B: Cobb method or Harrison posterior tangent method: which to choose for lateral cervical radiographic analysis. Spine (Phila Pa 1976) 25:2072-2078, 2000

11. Iyer S, Lenke LG, Nemani VM, Albert TJ, Sides BA, Metz $\mathrm{LN}$, et al: Variations in sagittal alignment parameters based on age: a prospective study of asymptomatic volunteers using full-body radiographs. Spine (Phila Pa 1976) 41:1826-1836, 2016
12. Iyer S, Lenke LG, Nemani VM, Fu M, Shifflett GD, Albert TJ, et al: Variations in occipitocervical and cervicothoracic alignment parameters based on age: a prospective study of asymptomatic volunteers using full-body radiographs. Spine (Phila Pa 1976) 41:1837-1844, 2016

13. Kuntz C IV, Levin LS, Ondra SL, Shaffrey CI, Morgan CJ: Neutral upright sagittal spinal alignment from the occiput to the pelvis in asymptomatic adults: a review and resynthesis of the literature. J Neurosurg Spine 6:104-112, 2007

14. Lafage R, Challier V, Liabaud B, Vira S, Ferrero E, Diebo BG, et al: Natural head posture in the setting of sagittal spinal deformity: validation of chin-brow vertical angle, slope of line of sight, and McGregor's slope with health-related quality of life. Neurosurgery 79:108-115, 2016

15. Moon BJ, Smith JS, Ames CP, Shaffrey CI, Lafage V, Schwab F, et al: Prevalence and type of cervical deformities among adults with Parkinson's disease: a cross-sectional study. J Neurosurg Spine 24:527-534, 2016

16. Passias PG, Oh C, Jalai CM, Worley N, Lafage R, Scheer JK, et al: Predictive model for cervical alignment and malalignment following surgical correction of adult spinal deformity. Spine (Phila Pa 1976) 41:E1096-E1103, 2016

17. Protopsaltis T, Bronsard N, Soroceanu A, Henry JK, Lafage R, Smith J, et al: Cervical sagittal deformity develops after PJK in adult thoracolumbar deformity correction: radiographic analysis utilizing a novel global sagittal angular parameter, the CTPA. Eur Spine J [epub ahead of print], 2016

18. Scheer JK, Tang JA, Smith JS, Acosta FL Jr, Protopsaltis TS, Blondel B, et al: Cervical spine alignment, sagittal deformity, and clinical implications: a review. J Neurosurg Spine 19:141-159, 2013

19. Shamji MF, Mohanty C, Massicotte EM, Fehlings MG: The association of cervical spine alignment with neurologic recovery in a prospective cohort of patients with surgical myelopathy: analysis of a series of 124 cases. World Neurosurg 86:112-119, 2016

20. Toyama Y, Matsumoto M, Chiba K, Asazuma T, Suzuki N, Fujimura Y, et al: Realignment of postoperative cervical kyphosis in children by vertebral remodeling. Spine (Phila Pa 1976) 19:2565-2570, 1994

21. von Elm E, Altman DG, Egger M, Pocock SJ, Gøtzsche PC, Vandenbroucke JP: The Strengthening the Reporting of Observational Studies in Epidemiology (STROBE) statement: guidelines for reporting observational studies. Ann Intern Med 147:573-577, 2007

\section{Disclosures}

The authors report no conflict of interest concerning the materials or methods used in this study or the findings specified in this paper.

\section{Author Contributions}

Conception and design: Bartels, Donk, Fehlings. Acquisition of data: Bartels, Arnts. Analysis and interpretation of data: Bartels, Donk, Verhagen, Arnts, Verbeek. Drafting the article: Bartels, Donk, Fehlings, Verhagen. Critically revising the article: all authors. Reviewed submitted version of manuscript: Bartels, Donk, Fehlings, Verhagen, Arnts, Verbeek. Approved the final version of the manuscript on behalf of all authors: Bartels. Statistical analysis: Groenewoud. Administrative/technical/material support: Verbeek. Study supervision: Bartels.

\section{Correspondence}

Ronald H. M. A. Bartels, Department of Neurosurgery, Radboud University Medical Center, Geert Groote Plein-zuid 10, 6525 GA Nijmegen, The Netherlands. email: ronald.bartels@radboudumc.nl. 